\title{
Alternative viscous-plastic rheologies for the representation of fracture lines in high-resolution sea ice models
}

Damien Ringeisen, Martin Losch, and L. Bruno Tremblay

$$
\text { vEGU } 2021 \text { - EGU21-1373 - 2021-04-30 }
$$

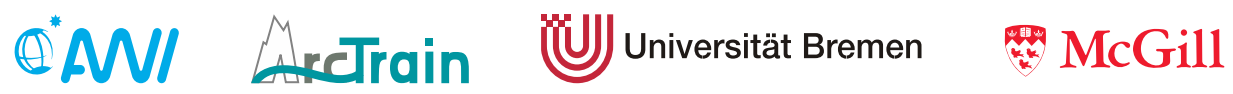


A little thought experiment...

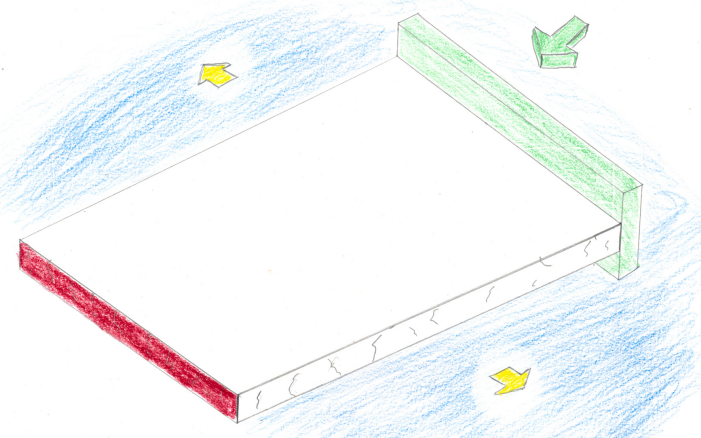

@ $\odot$ 


\section{A little thought experiment...}

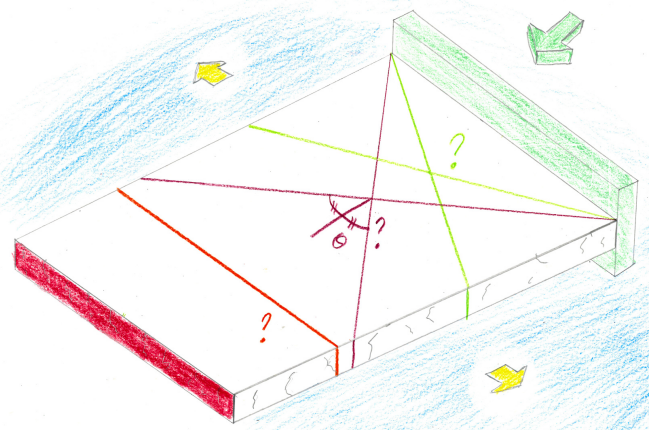


A little thought experiment... 
A little thought experiment... 


\section{... which we can observe on the field.}

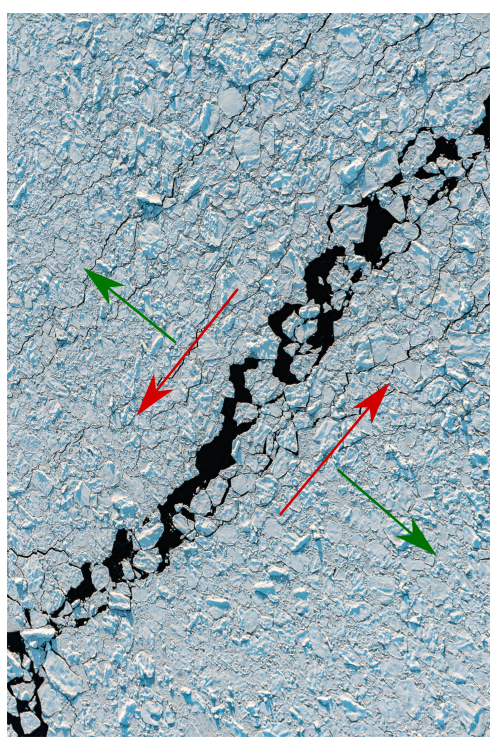

Credit: Lukas Piotrowski

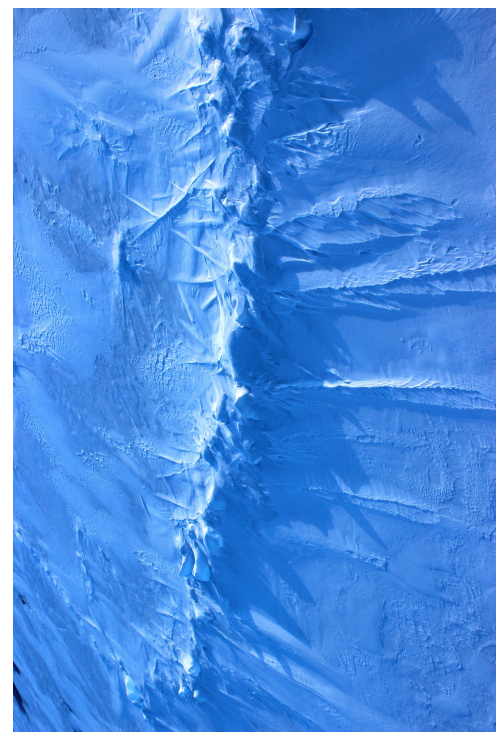

Credit: Grace Shephard (distributed via imaggeo.egu.eu) CC-BY-NC 


\section{Motivation?}

The overarching motivation

September Arctic Sea Ice Volume (PIOMAS)

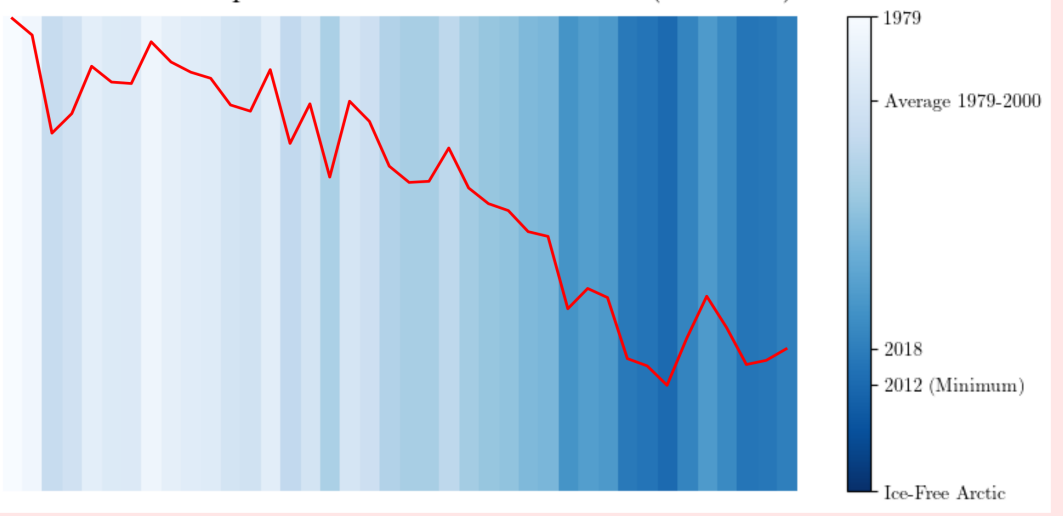




\section{Motivation?}

We call these deformation lines Linear Kinematic Features or LKFs.

\section{LKFs influence}

- Exchange of Energy and Moisture

- Creation of new ice $\rightarrow$ in leads

- Creation of thick ice $\rightarrow$ in ridges

$\rightarrow$ Influence the mass balance

\section{We}

- Observe the LKFs intersection angles in deformation patterns

- Want to reproduce these patterns in sea ice dynamical models

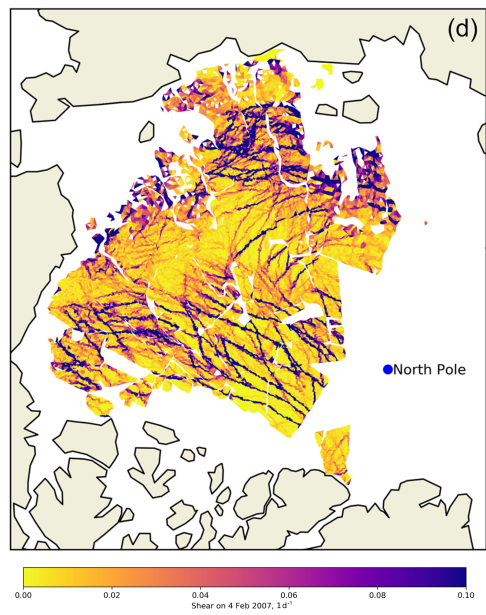

Figure: Shear Deformation - From Rampal et al. (2019) - under CC-BY license. 


\section{The sea ice Viscous-Plastic (VP) rheological model}

The most widely used sea ice model

n Viscous for small deformations $\rightarrow$ Plastic for large deformations (Hibler, 1977)

- Two main components:

\section{A yield curve}

- Transition between Viscous and Plastic in the stress space

- Viscous deformation are slow $\left(t_{\text {def }} \simeq 35 y\right)$

- Almost a purely plastic model

\section{A flow rule}

- Post-failure deformation

- i.e. the ratio of shear and divergence or convergence

- Can be normal or non-normal to the yield curve

We call rheology the coupling of a yield curve shape and a flow rule.

VP was designed for resolution of $\mathrm{O}(100 \mathrm{~km})$ and is now used at resolution of $\mathrm{O}(1 \mathrm{~km})$ 


\section{Models and observation disagreen on LKFs intersection angles}

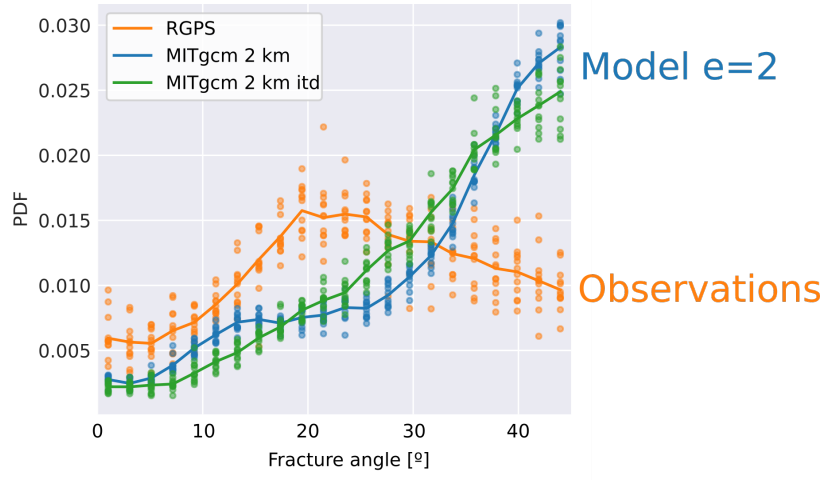

Figure: PDFs of LKFs half-intersection angles — Derived from Hutter and Losch (2020) - under CC-BY license.

See the work of Nils Hutter on comparing sea ice rheological model here at vEGU21: EGU21-9739 


\section{Theory of fracture angles in granular matter}

- Coulomb Angle $\theta_{C}$ (Coulomb, 1773):

The fracture angle depends on the slope of the yield curve, i.e., the stress ratio $\phi$ along the shear line.

- Roscoe Angle $\theta_{R}$ (Roscoe, 1970):

The fracture angle depends on the orientation of the flow rule, i.e., the strain-rate ratio $\delta$ along the shear line.

- Arthur Angle $\theta_{A}$ (Arthur et al., 1977):

The fracture angle is the average of $\theta_{C}$ and $\theta_{R}$.

$\rightarrow$ with a normal flow rule, then $\theta_{C}=\theta_{R}=\theta_{A}$

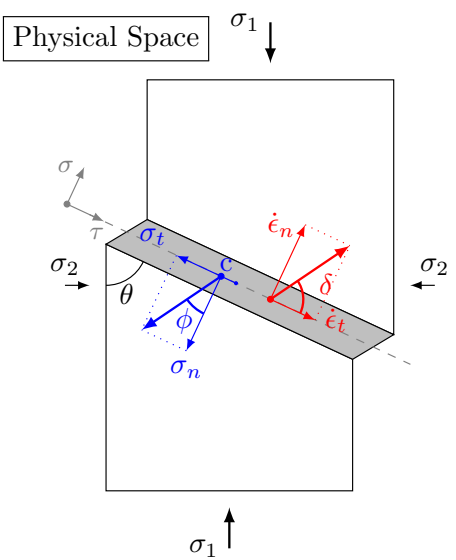




\section{Experimental setup: Uni-axial compression}
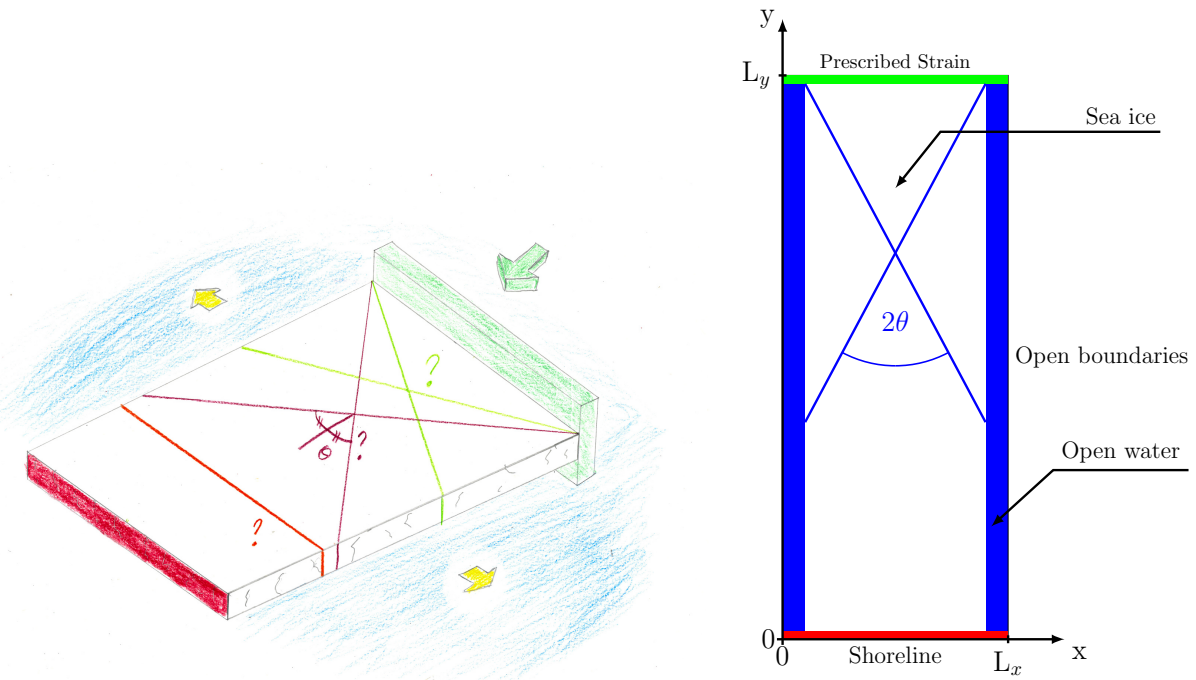


\section{Recent results with the same setup}

Ringeisen et al. (2019)

- Ellitptical yield curve with normal flow rule (Hibler, 1979)

- Fracture angles depend on the yield curve slope with a normal flow rule

- Cannot create angles smaller than $30^{\circ}$ in uni-axial compression
Ringeisen et al. (2020)

- Designed a elliptical yield curve with non-normal flow rule.

- The direction of the flow rule sets the fracture angle $\rightarrow$ Roscoe angle

- Able to create angles smaller than $30^{\circ}$ in uni-axial compression

\section{Here we}

- Investigate yield curves that do not have an elliptical shape.

- Especially Mohr-Coulomb yield curve, known for the modelling granular materials.

- Insist on good numerical convergence to explore the precise effects of the rheology.

- Idealized compression experiment

with the MITgcm sea ice package (Losch et al., 2010). 


\section{New yield curves: Mohr-Coulomb \& Teardrop}

Mohr-Coulomb yield curve (MCE) non-normal flow rule

derived from Ip et al. (1991)

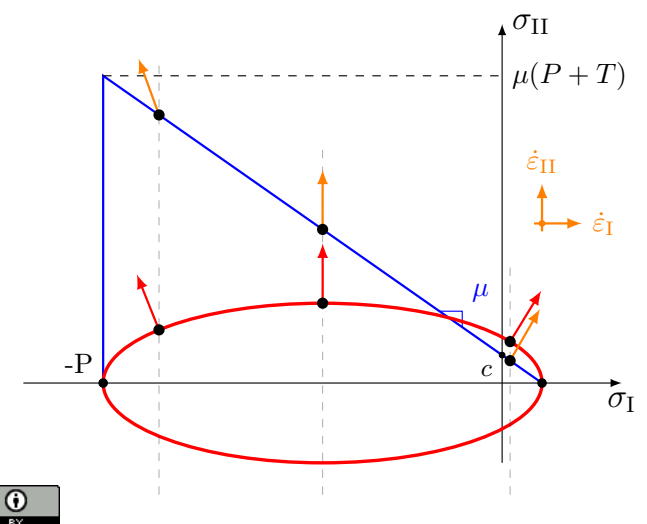

Teardrop yield curve (TD) normal flow rule

modified from Zhang and Rothrock (2005)

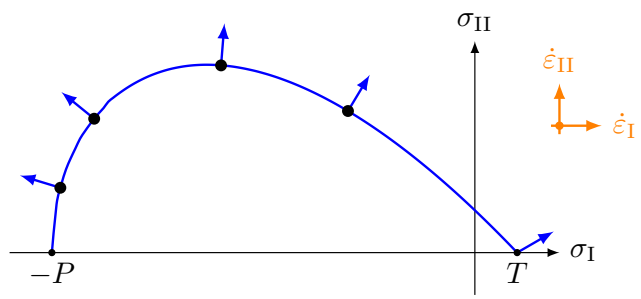




\section{Results: Mohr-Coulomb yield curve}

- Creates defined shear lines, unlike the formulation of Ip et al. (1991).
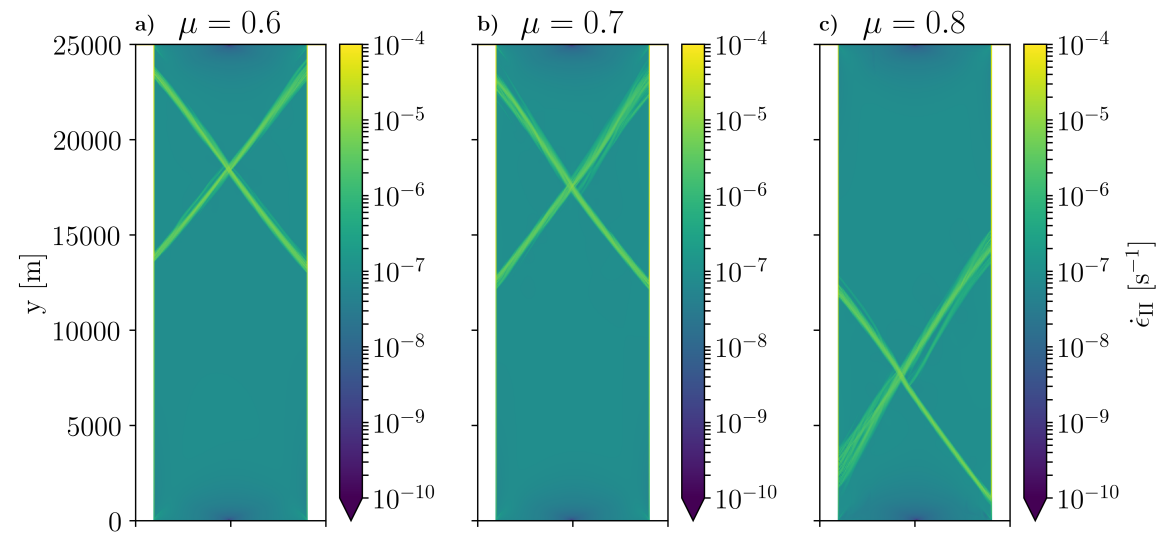


\section{Results: Mohr-Coulomb yield curve}

- Creates defined shear lines, unlike the formulation of Ip et al. (1991).

- The fracture angles correspond to the Arthur angles.

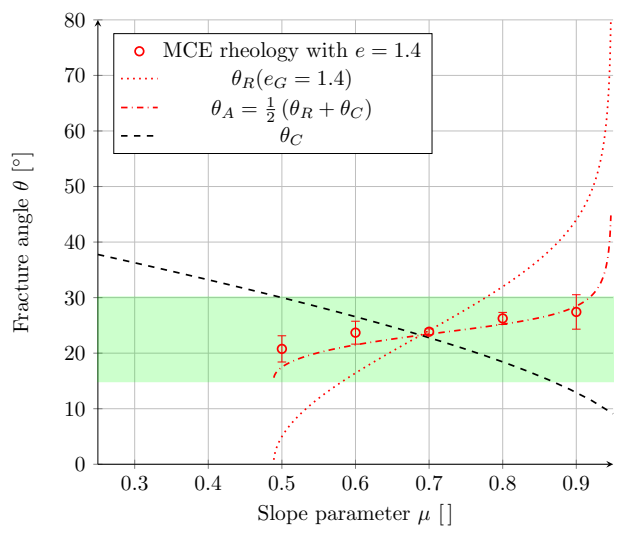




\section{Results: Mohr-Coulomb yield curve}

- Creates defined shear lines, unlike the formulation of Ip et al. (1991).

- The fracture angles correspond to the Arthur angles.
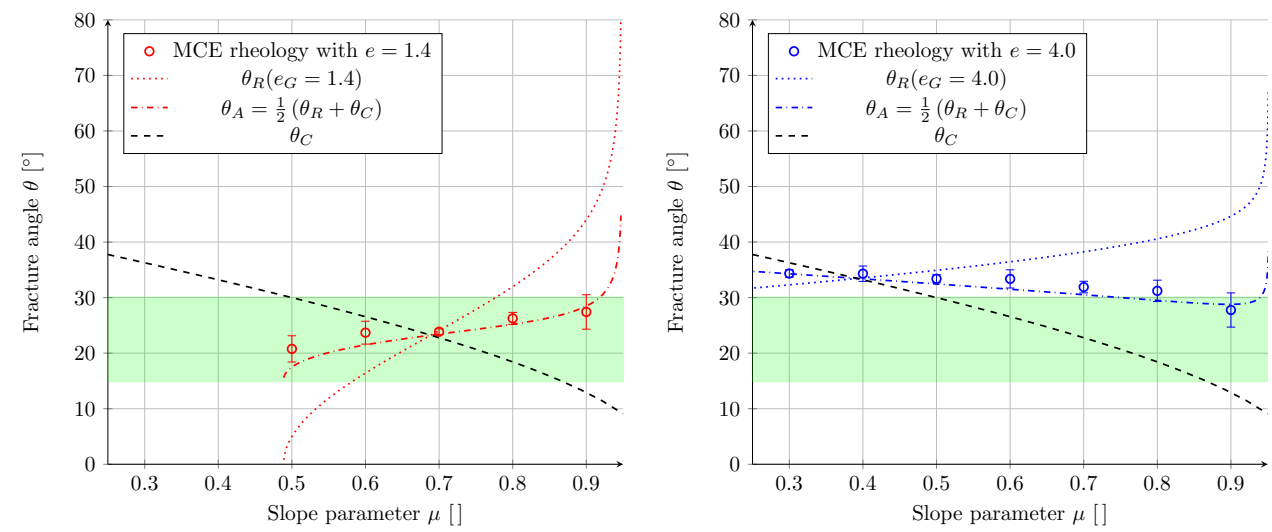


\section{Results: Teardrop yield curve}

- Creates defined shear lines with small angles.
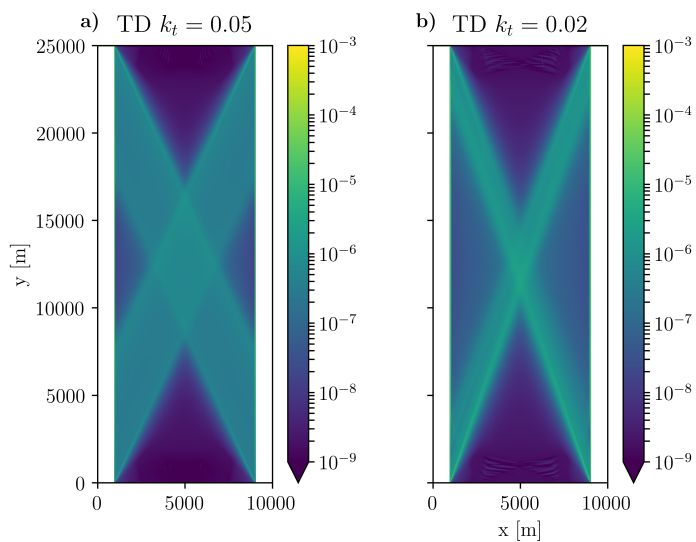


\section{Results: Teardrop yield curve}

- Creates defined shear lines with small angles.

- Modeled angles fit exactly the theoretical angles with normal flow rule.

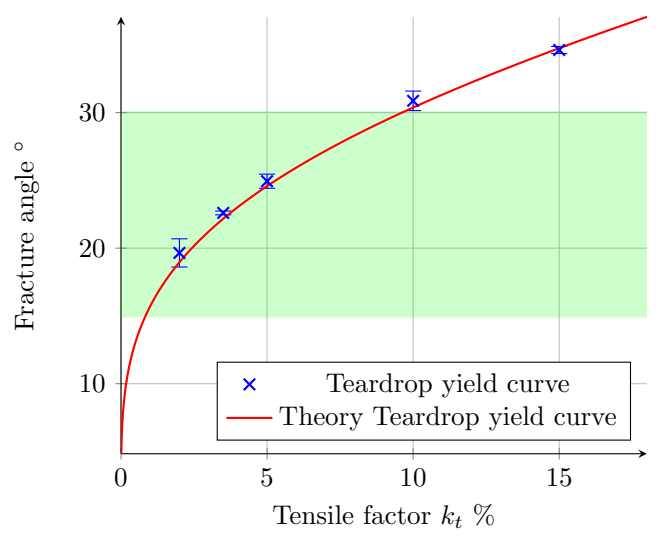




\section{Conclusions and Outlook}

\section{Mohr-Coulomb}

- Surprisingly: Shear lines with Arthur angles

- Contradicts our previous work (Ringeisen et al., 2020).

- Unknown reason yet.

- Allows to decrease the fracture angles. 


\section{Conclusions and Outlook}

\section{Mohr-Coulomb}

- Surprisingly: Shear lines with Arthur angles

- Contradicts our previous work (Ringeisen et al., 2020).

- Unknown reason yet.

- Allows to decrease the fracture angles.

\section{Teardrop}

- Very good agreement with theory

- Clean fracture pattern, with issues fixed.

- Also allows to decrease the angles

- Good candidate to reduce the fracture angles overall. 


\section{Conclusions and Outlook}

\section{Mohr-Coulomb}

- Surprisingly: Shear lines with Arthur angles

- Contradicts our previous work (Ringeisen et al., 2020).

- Unknown reason yet.

- Allows to decrease the fracture angles.

\section{Teardrop}

n Very good agreement with theory

- Clean fracture pattern, with issues fixed.

- Also allows to decrease the angles

- Good candidate to reduce the fracture angles overall.

\section{Conclusions}

- Essential to test our rheological models

- We can reduce the fracture angles with non-elliptical yield curves

- With a yield curve for granular properties 


\section{Conclusions and Outlook}

\section{Mohr-Coulomb}

- Surprisingly: Shear lines with Arthur angles

- Contradicts our previous work (Ringeisen et al., 2020).

- Unknown reason yet.

- Allows to decrease the fracture angles.

\section{Teardrop}

n Very good agreement with theory

- Clean fracture pattern, with issues fixed.

- Also allows to decrease the angles

- Good candidate to reduce the fracture angles overall.

\section{Conclusions}

- Essential to test our rheological models

- We can reduce the fracture angles with non-elliptical yield curves

- With a yield curve for granular properties

\section{Outlook}

- Yield curves implemented in the MITgcm sea ice package

- Currently testing their effect in high-resolution pan-Arctic simulations 


\section{Summary - Contact us for more info}

\section{Deformation lines in sea ice}

- Intersection angles are larger in models than observed.

- Linked to the Viscous-Plastic rheology

\section{Two modified rheologies}

- Mohr-Coulomb yield curve - non-normal

- Teardrop yield curve - normal flow rule

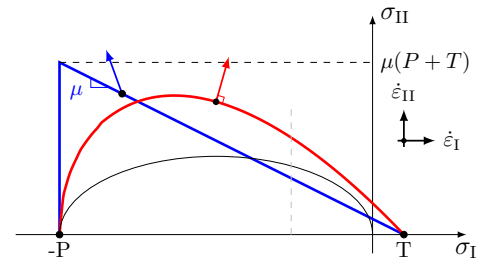

\section{Idealized numerical experiment}

- Both rheologies allow for smaller angles

- MCE creates fractures with Arthur angles

- Investigating rheologies is necessary

- Available in MITgcm now

n Next step: test in pan-arctic setups 


\section{References I}

Arthur, J. R. F., Dunstan, T., Al-Ani, Q. a. J. L., and Assadi, A. (1977). Plastic deformation and failure in granular media. Géotechnique, $27(1): 53-74$.

Coulomb, C. (1773). Test on the applications of the rules of maxima and minima to some problems of statics related to architecture. Mem Math Phys, 7:343-382.

Hibler, W. D. (1977). A viscous sea ice law as a stochastic average of plasticity. Journal of Geophysical Research, 82(27):3932-3938.

Hibler, W. D. (1979). A Dynamic Thermodynamic Sea Ice Model. Journal of Physical Oceanography, 9(4):815-846.

Hutter, N. and Losch, M. (2020). Feature-based comparison of sea ice deformation in lead-permitting sea ice simulations. The Cryosphere, 14(1):93-113.

Ip, C. F., Hibler, W. D., and Flato, G. M. (1991). On the effect of rheology on seasonal sea-ice simulations. Annals of Glaciology, $15: 17-25$.

Losch, M., Menemenlis, D., Campin, J.-M., Heimbach, P., and Hill, C. (2010). On the formulation of sea-ice models. Part 1: Effects of different solver implementations and parameterizations. Ocean Modelling, 33(1-2):129-144.

Rampal, P., Dansereau, V., Olason, E., Bouillon, S., Williams, T., Korosov, A., and Samaké, A. (2019). On the multi-fractal scaling properties of sea ice deformation. The Cryosphere, 13(9):2457-2474. Publisher: Copernicus GmbH.

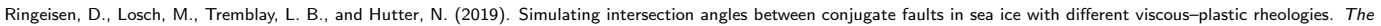
Cryosphere, 13(4):1167-1186.

Ringeisen, D., Tremblay, L. B., and Losch, M. (2020). Non-normal flow rules affect fracture angles in sea ice viscous-plastic rheologies. The Cryosphere Discussions.

Roscoe, K. H. (1970). The Influence of Strains in Soil Mechanics. Géotechnique, 20(2):129-170.

Zhang, J. and Rothrock, D. A. (2005). Effect of sea ice rheology in numerical investigations of climate. Journal of Geophysical Research: Oceans, 110(C8):C08014. 